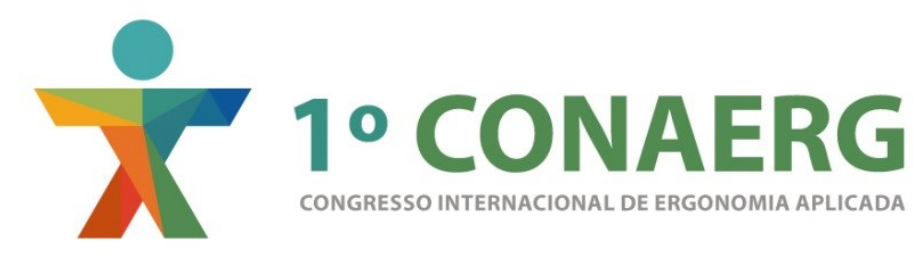

\title{
FUTURO DO TRABALHO COM O USO DO COMPUTADOR PESSOAL: ANÁLISE DAS EXIGÊNCIAS ERGONÔMICAS NO USO DO COMPUTADOR DE MESA E LAPTOP
}

\author{
Fabíola Reinert (1); \\ Raoni Pontes Caselli (2); \\ Antônio Renato Pereira Moro (3); \\ Leila Amaral Gontijo (4); \\ Marcelo Gitirana Gomes Ferreira (5) \\ (1) Universidade Federal de Santa Catarina, Doutoranda \\ e-mail:fabiola.reinert@gmail.com \\ (2) Universidade Federal de Santa Catarina, Mestrando \\ e-mail:raonicaselli@gmail.com \\ (3) Universidade Federal de Santa Catarina, Dr. \\ e-mail:renato.moro@ufsc.br \\ (4) Universidade Federal de Santa Catarina, Dr. \\ e-mail:leila.gontijo@ufsc.br \\ (5) Universidade Federal de Santa Catarina, Dr. \\ e-mail:marcelo.gitirana@gmail.com
}

\begin{abstract}
RESUMO
O objetivo deste estudo foi determinar as exigências ergonômicas no uso de computadores de mesa e laptops. Para efeitos de comparação foram verificadas as posturas no plano sagital e transversal envolvidas durante a execução de uma tarefa de digitação e edição em um computador de mesa e em um laptop. Sendo assim, foi realizado um estudo de caso com um indivíduo brasileiro de estatura mediana, com observação direta e filmagem. Concluiu-se que o uso de computadores de mesa apresenta menor exigência biomecânica, pois apresentou ângulos mais próximos de uma posição neutra e consequentemente um menor risco de queixas musculoesqueléticas.
\end{abstract}

\begin{abstract}
The aim of this study was to determine the ergonomic requirements in the use of desktop computers and laptops. For comparison, postures in the sagittal and transversal planes involved while performing a typing and editing task on a desktop and laptop computers were verified. Thus, a case study was performed with direct observation and filming of a Brazilian male of medium height. It is concluded that the use of desktop computers has lesser biomechanical demands since it showed angles closer to a neutral position and consequently having a lower risk of musculoskeletal complaints.
\end{abstract}




\section{INTRODUÇÃO}

Ao longo dos últimos anos, uma extensa pesquisa foi realizada sobre as posturas mais adequadas a adotar durante o uso de computadores (VILLANUEVA et al., 1996; STRAKER et al., 1997; SOMMERICH et al., 2002; SEGHERS et al., 2003). Relatos na literatura científica sugerem que usuários de computadores têm um maior risco de queixas musculoesqueléticas dos membros superiores (HOROWITZ, 1992; TITTIRANONDA et al., 1999), e, devido à sua ampla utilização, mesmo riscos relativamente pequenos associados à utilização de computadores têm importantes implicações para a saúde pública (GERR et al., 2004).

O uso do computador é comumente relacionado ao aparecimento de queixas musculoesqueléticas (DONOGHUE et al., 2013, AYDENIZ; GURSOY, 2008; CONLON; REMPEL, 2005). Punnett e Bergqvist (1997) identificaram os seguintes fatores de exigências biomecânicas no uso do computador: desvio ulnar do punho, extensão do punho, flexão de cotovelo, flexão de ombro, bem como flexão e rotação do pescoço. Geralmente, posturas não neutras são considerados como prejudiciais (GOLD et al., 2012). Gerr et al. (2006) afirma que as posturas adotadas no uso do computador de mesa são diferentes das adotadas no uso de computadores portáteis.

Atualmente, a portabilidade permitida pelos mini-computadores fez com que os volumes de vendas destes excedessem muito as dos computadores de mesa (GOLD et al., 2012). Em 2008 , as vendas de laptops superaram as vendas trimestrais de computadores de mesa com mais de 9,5 milhões de unidades vendidas (MANN, 2008). De acordo com Straker et al. (1997) os computadores portáteis foram introduzidas no mercado de trabalho por razões de portabilidade, e foram projetados apenas para uso em curtos períodos de tempo, em situações que impossibilitavam a utilização de computadores de mesa.

Os computadores portáteis podem ser usados em uma variedade de posturas quando não acoplado à estação de trabalho (GOLD et al., 2012), entretanto o aumento da sua portabilidade também aumenta a exposição à potenciais fatores de risco por queixas musculoesqueléticas em relação aos computadores de mesa (ASUNDI et al., 2012). Em particular, uma vez que o visor e teclado são ligados, a altura do ecrã é normalmente inferior à recomendada (STRAKER et al., 1997; BERKHOUT et al., 2004; ASUNDI et al., 2012).

Usuários de computadores portáteis relataram maiores constrangimentos posturais e atividades musculares do pescoço do que os de computadores de mesa. As queixas de desconforto ocular e musculoesqueléticos, bem como dificuldade de digitação foram maiores durante o trabalho com os computadores portáteis (JONAl et al., 2002). Em comparação com computadores de mesa, estudos mostram que o uso de computadores portáteis resulta em maior flexão do pescoço e inclinação da cabeça (STRAKER et al., 1997; SOMMERICH et al., 2002; SEGHERS et al., 2003) e uma maior atividade de extensão do pescoço (SAITO et al., 1997; VILLANUEVA et al., 1998; SEGHERS et al., 2003).

Sendo assim, considerando as questões ergonômicas envolvidas no uso dos computadores, o objetivo deste estudo foi determinar se existem diferentes fatores de natureza ergonômica associados à evolução dos computadores, verificando as posturas e angulações corporais envolvidas no uso dos computadores de mesa e laptops, além das intensidades de desconforto em cada caso.

Atualmente os computadores portáteis vêm substituindo os computadores de mesa em ambientes de trabalho, e com a rápida evolução tecnológica, os produtos ficam cada dia menores e mais portáteis. Devido ao aumento do número de trabalhos em escritórios ou homeoffice, em relação à industria, tem-se a necessidade de estudos que beneficiem esses profissionais, e que permitam compreender e analisar o futuro do trabalho no sentido de impedir ou amenizar riscos ergônomicos. 


\section{MATERIAIS E MÉTODOS}

Trata-se de uma pesquisa de caráter exploratória-descritiva, tendo como objetivo detectar, compreender e interpretar o fenômeno investigado (GIL, 1996; TRIVINÕS, 2006; CERVO et al., 2007). Para tanto, foi realizado um estudo de caso, que se caracteriza como um tipo de pesquisa cujo objeto é uma unidade que se analisa aprofundadamente (TRIVINÕS, 2006). Sendo assim, foram analisadas as posições adotadas pelo sujeito no uso do computador de mesa e laptop, por meio de observação direta e filmagem, utilizando a técnica de videografia. Para a análise das imagens utilizou-se o software Kinovea e Corel Draw, e o programa Excel para compilação de dados.

O projeto em questão contemplou os procedimentos descritos pelo Conselho Nacional de Saúde, sob Resolução 196-1996 (BRASIL, 1996) e pela Norma ERG-BR 1002 do Código de Deontologia do Ergonomista Certificado (ABERGO, 2002), atendendo às exigências éticas e científicas fundamentais. Para tanto, foi aplicado um TCLE - Termo de Consentimento Livre e Esclarecido, o sujeito preencheu um protocolo com informações pessoais básicas e foi utilizado outro protocolo, sem identificação do sujeito, para coleta dos dados.

O sujeito analisado é do sexo masculino, tem 29 anos e trabalha em torno de $20 \mathrm{~h} / \mathrm{semana}$ com computador, sendo que atualmente faz uso de um Laptop para trabalhar. O indivíduo mede 1,70 de altura e tem $62 \mathrm{~kg}$, considerando que de acordo com lida (2005) a estatura média do brasileiro é de $1,70 \mathrm{~m}$ e o peso $60 \mathrm{~kg}$, o sujeito foi considerado como padrão mediano. O sujeito não apresentava distúrbios musculoesqueléticos prévios ao estudo.

Para a realização do estudo foram consideradas 3 situações diferenciadas: (a) uso do computador de mesa acoplado a estação de trabalho, (b) uso do laptop acoplado a estação de trabalho, (c) uso do laptop em poltrona disponível no ambiente de trabalho. Foi utilizado um computador de mesa da marca LG, e um laptop ASUS 546C.

O método de abordagem foi análise documental e observação direta utilizando a técnica de Videografia. Para a videografia foi utilizada uma câmera Nikon L110 posicionada a $1 \mathrm{~m}$ do participante, paralela ao plano sagital, e uma câmera Fujifilm S4200, posicionada a $0,5 \mathrm{~m}$ do participante, paralela ao plano transversal. A estação de trabalho consistiu em uma mesa de $69 \times 170 \mathrm{~cm}$ e uma cadeira ajustável, sendo que o sujeito foi instruído à ajustar a cadeira para uma altura confortável.

Aderiram-se nove marcadores esféricos de isopor de $2,5 \mathrm{~cm}$ de diâmetro com fita adesiva de duplo contato do lado direito do participante do estudo. Para fins de melhor identificar os ângulos posturais, os marcadores foram situados em: (1) Margem lateral do olho, (2) Atrás da orelha, (3) Processo espinhoso de C7, (4) Articulação acromio-clavicular, (5) Epicôndilo lateral do húmero, (6) Processo estilóide do cúbito, (7) Cabeça do quinto metacarpiano, (8) Ponto médio do trocánter maior do fêmur, (9) Centro da tela do laptop no plano sagital.

A partir dos nove marcadores e as linhas de referência horizontal e vertical, foram obtidos os nove ângulos posturais (Figura 1) definidos da seguinte forma (CASTELLUCCI; ZÚÑIGA BENITEZ, 2013):

1. Ângulo de visão: ângulo formado pela linha que vai desde a margem lateral do olho até o centro da tela, com respeito a linha horizontal. Designa-se valor zero a linha de referência horizontal. Inferior a esta o valor do ângulo é negativo e superior a esta, positivo.

2. Inclinação da cabeça: ângulo formado pela linha que vai desde a margem lateral do olho até atrás da orelha com respeito à linha de referência horizontal. Designa-se valor zero a linha horizontal. Inferior a esta o valor do ângulo é negativo e superior a esta, positivo.

3. Flexão do pescoço: ângulo formando pela linha que vai desde atrás da orelha até o processo espinhoso de C7, com respeito a linha de referência vertical. Designa-se 
valor zero a linha vertical. Anterior a esta o valor de ângulo é positivo e posterior a esta, negativo.

4. Ângulo craneo-cervical: ângulo formando pela linha que vai desde a margem externa do olho até atrás da orelha e a linha que vai desde este último até o processo espinhoso de C7.

5. Ângulo cérvico-torácico: ângulo formado pela linha que vai desde atrás da orelha até o processo espinhoso de $\mathrm{C} 7$ e da linha que vai desde este último até o ponto médio do trocanter maior do fêmur.

6. Flexão de ombro: ângulo formando pela linha que vai desde a articulação acromioclavicular até o epicôndilo lateral do húmero, com respeito a linha de referência vertical. Designa-se valor zero a linha vertical. Anterior a esta o valor do ângulo é positivo e posterior a este negativo.

7. Flexão de cotovelo: ângulo formado pela linha que vai desde a articulação acromioclavicular até o epicôndilo lateral do húmero, e a linha deste último até o processo estilóide do cúbito. $\mathrm{O}$ valor deste ângulo é progressivamente positivo e o sentido inverso dos ponteiros do relógio.

8. Flexão-extensão de punho: ângulo formado pela linha que vai desde o epicôndilo lateral do húmero até o processo estilóides do cúbito e a linha que vai desde este último até a cabeça do quinto metacarpiano. Designa-se valor zero a primeira linha mencionada. Inferior a esta o valor do ângulo é negativo e superior a esta, positivo.

9. Inclinação do tronco: ângulo formando pela linha que vai desde o processo espinhoso de $\mathrm{C} 7$ até o ponto médio do trocánter maior do fêmur, com respeito à linha de referência horizontal.

10. Desvio radial-ulnar do punho: ângulo formado pela linha que vai desde o epicôndilo lateral do húmero até o processo estilóides do cúbito e a linha que vai desde este último até a cabeça do quinto metacarpiano, no plano transversal. Designa-se valor zero a primeira linha mencionada. Inferior a esta o valor do ângulo é negativo e superior a esta, positivo.

Figura 1- Fotos do sujeito no plano sagital e frontal e a identificação dos pontos de referência anatômicos, com os respectivos ângulos de interesse: 1. ângulo da visão, 2. inclinação da cabeça, 3. flexão do pescoço, 4. ângulo craneo-cervical, 5. ângulo cérvico-torácico, 6 . flexão de ombro, 7. flexão de cotovelo, 8. flexão-extensão de pulso, 9. inclinação do tronco, 10. desvio radial-ulnar do punho.

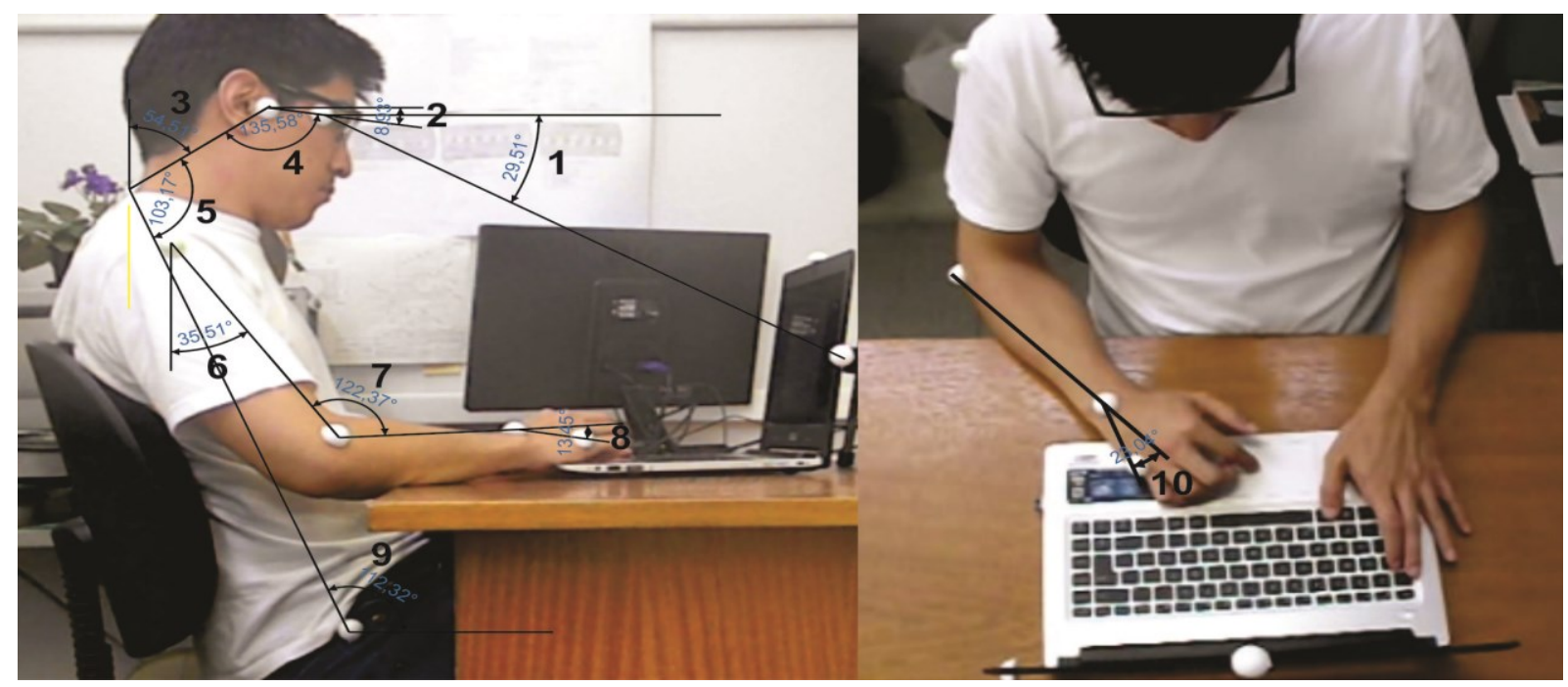

Fonte: os autores. 
Foram filmadas as atividades de trabalho do indivíduo estudado, que envolvem digitação e edição de texto, durante aproximadamente 15 minutos, sendo que foram analisados os 5 minutos intermediários. As abordagens foram conduzidas dentro do ambiente de trabalho, durante a prática da atividade, sendo realizadas 3 abordagens em dias distintos, no período matutino, antes de qualquer interação com computador naquele dia.

\section{RESULTADOS E DISCUSSÃO}

Os resultados das posturas assumidas durante a condição $\mathrm{N}^{\circ} 1$ (uso do computador de mesa), condição $\mathrm{N}^{\circ} 2$ (uso do Laptop), e condição $\mathrm{N}^{\circ} 3$ (uso do Laptop na poltrona) (Figura 2) podem ser verificadas na Tabela 1. Foram analisadas 60 imagens dos vídeos de cada condição para gerar os dados necessários para o estudo dos ângulos posturais.

Figura 2 - Fotos do sujeito nas três diferentes situações analisadas: acima, condição №1 (uso do computador de mesa), no meio, condição $N^{\circ} 2$ (uso do laptop) e abaixo, condição $N^{\circ} 3$ (uso do laptop na poltrona).

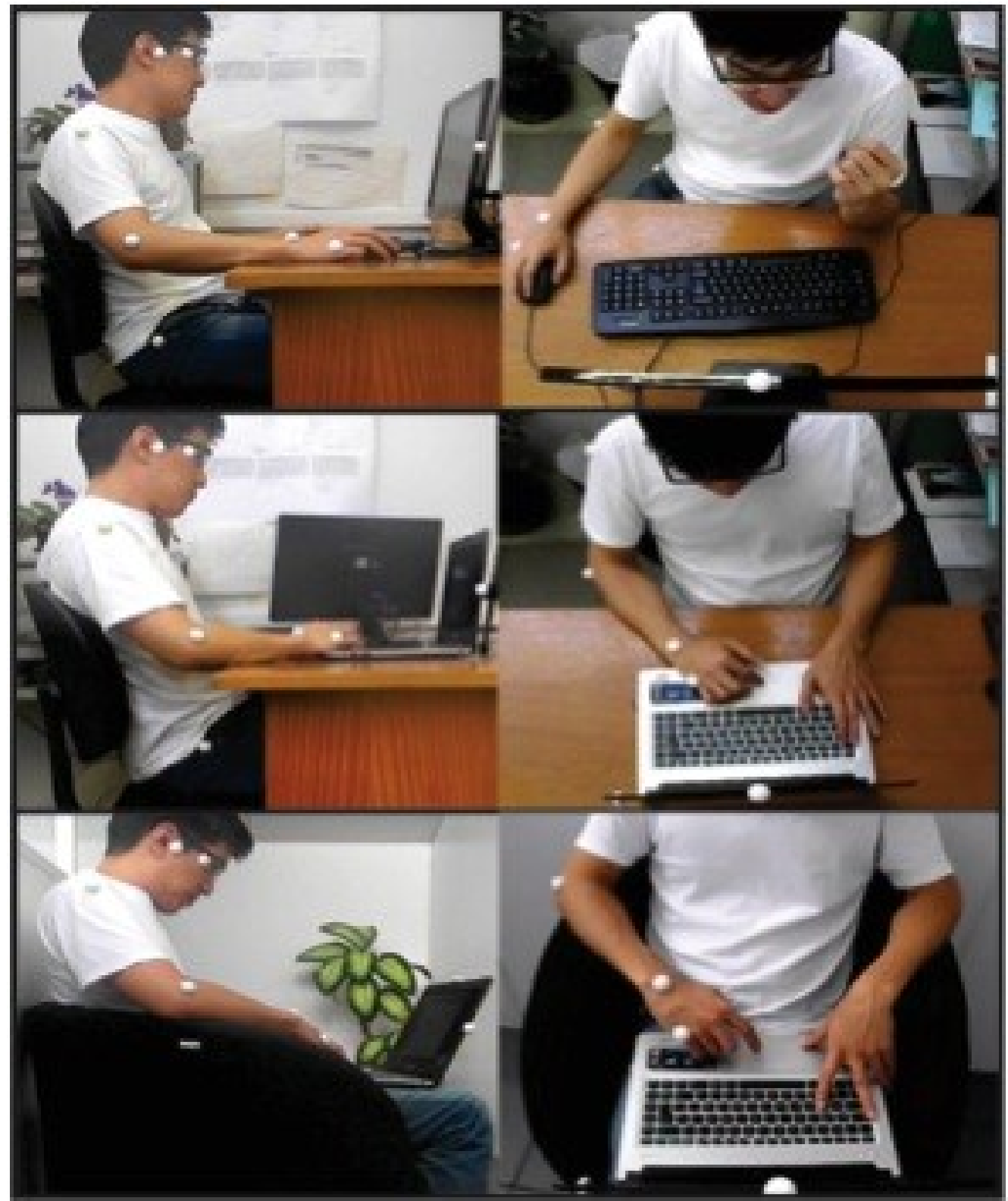

Fonte: os autores. 
Tabela 1 - Resumo de análises das posturas assumidas nas três condições.

\begin{tabular}{|c|c|c|c|c|c|c|}
\hline \multirow{2}{*}{$\begin{array}{l}\text { Ângulos } \\
\text { Posturais }\end{array}$} & \multicolumn{2}{|c|}{ Condição $\mathrm{N}^{\circ} 1\left(^{\circ}\right)$} & \multicolumn{2}{|c|}{ Condição $\mathrm{N}^{\circ} 2\left(^{\circ}\right)$} & \multicolumn{2}{|c|}{ Condição No3 ( $\left.{ }^{\circ}\right)$} \\
\hline & Média & DP & Média & DP & Média & DP \\
\hline 1 & $-21,18$ & 5,51 & $-28,49$ & 6,83 & $-36,85$ & 5,50 \\
\hline 2 & 1,75 & 7,21 & $-9,57$ & 9,06 & $-29,12$ & 7,69 \\
\hline 3 & 47,42 & 10,43 & 57,79 & 8,95 & 75,09 & 8,53 \\
\hline 4 & 142,86 & 8,39 & 132,84 & 6,12 & 130,73 & 8,12 \\
\hline 5 & 117,40 & 7,41 & 104,36 & 6,19 & 99,53 & 8,45 \\
\hline 6 & 24,78 & 9,84 & 37,79 & 12,83 & 40,18 & 12,58 \\
\hline 7 & 106,79 & 16,99 & 122,52 & 14,97 & 143,42 & 16,43 \\
\hline 8 & $-8,12$ & 11,08 & $-13,74$ & 12,32 & $-28,63$ & 12,30 \\
\hline 9 & 102,34 & 8,92 & 114,19 & 9,67 & 98,41 & 8,44 \\
\hline 10 & 18,52 & 10,92 & 24,35 & 9,19 & 28,14 & 9,34 \\
\hline
\end{tabular}

Fonte: os autores.

De acordo com os resultados encontrados, pode-se observar que a condição experimental $\mathrm{N}^{\circ} 1$ apresentou em relação às outras duas condições menor valor no: ângulo de visão (condição $\mathrm{N}^{\circ}$ 2: diferença de $7,31^{\circ}$; condição $\mathrm{N}^{\circ} 3$ : diferença de $15,67^{\circ}$ ), inclinação da cabeça (condição $\mathrm{N}^{\circ}$ 2: diferença de $11,32^{\circ}$ e condição $\mathrm{N}^{\circ} 3$ : diferença de $30,87^{\circ}$ ), flexão do pescoço (condição $\mathrm{N}^{\circ} 2$ : diferença de $10,37^{\circ}$ e condição $\mathrm{N}^{\circ} 3$ : diferença de $27,67^{\circ}$ ), flexão de ombro (condição $\mathrm{N}^{\circ} 2$ : diferença de $13,01^{\circ}$ e condição $\mathrm{N}^{\circ} 3$ : diferença de $15,4^{\circ}$ ), flexão de cotovelo (condição $\mathrm{N}^{\circ} 2$ : diferença de $15,73^{\circ}$ e condição $\mathrm{N}^{\circ} 3$ : diferença de $36,63^{\circ}$ ), flexão-extensão do punho (condição $\mathrm{N}^{\circ} 2$ : diferença de $5,62^{\circ}$ e condição $\mathrm{N}^{\circ} 3$ : diferença de $20,51^{\circ}$ ) e desvio radial-ulnar do punho (condição $\mathrm{N}^{\circ} 2$ : diferença de $5,83^{\circ}$ e condição $\mathrm{N}^{\circ} 3$ : diferença de $9,62^{\circ}$ ). Os ângulos crâneo-cervical e cérvico-torácico foram maiores na condição experimental $\mathrm{N}^{\circ} 1$ (Ângulo crâneo-cervical: condição $\mathrm{N}^{\circ} 2$ : diferença de $10,02^{\circ}$ e condição $\mathrm{N}^{\circ} 3$ : diferença de 12,13; Ângulo cérvico-torácico: condição $\mathrm{N}^{\circ} 2$ : diferença de $13,04^{\circ}$ e condição $\mathrm{N}^{\circ} 3$ : diferença de $17,87^{\circ}$ ) enquanto a inclinação do tronco foi maior na condição $\mathrm{N}^{\circ} 2$ (condição $\mathrm{N}^{\circ}$ 1: diferença de $11,85^{\circ}$ e condição $\mathrm{N}^{\circ} 3$ : diferença de $15,78^{\circ}$ ).

Esses valores demonstram que a condição experimental $\mathrm{N}^{\circ} 1$ realizada com um computador de mesa, em relação às demais condições experimentais, se traduz em uma postura mais erguida da cabeça e do pescoço e uma postura mais neutra dos ombros, cotovelos e punho. Nota-se também que a condição experimental $\mathrm{N}^{\circ} 2$, realizada com um laptop em uma mesa de trabalho, apresentou valores mais próximos ao de uma postura neutra em comparação com a condição experimental $\mathrm{N}^{\circ} 3$, realizada com um laptop em uma poltrona, em todos os ângulos posturais exceto os ângulos crâneo-cervical, cérvico-torácico e a inclinação do tronco.

Os resultados encontrados nesse estudo foram semelhantes aos resultados obtidos em outros estudos (CASTELLUCCI; ZÚÑIGA BENITEZ, 2013), demonstrando que os fatores mais impactantes nos ângulos posturais entre o uso de um computador de mesa e um laptop são a altura do monitor e o uso de componentes externos (teclado e mouse). Castellucci e Zúñiga Benitez (2013) obtiveram os seguintes valores para ângulo de visão, inclinação da cabeça, flexão de pescoço, ângulo craneo-cervical, ângulo cérvico-torácico, flexão de ombro, flexão de cotovelo, flexão-extensão de pulso e inclinação do tronco, respectivamente: $-40,3^{\circ} ;-4,64^{\circ} ; 61,42^{\circ} ; 147,13^{\circ} ; 113,44^{\circ} ; 20,87^{\circ} ; 94,66^{\circ} ;-11,59^{\circ} ; 94,23^{\circ}$ em experimento com laptop acoplado a uma estação de trabalho, obtendo melhores resultados com o laptop utilizando componentes externos como teclado e mouse. 
Assim como nesse estudo se obteve $-9,57^{\circ}$ para inclinação da cabeça no uso do laptop e $1,75^{\circ}$ no uso do computador de mesa, Straker et al. (1997) obteve os valores $-9,8^{\circ}$ para inclinação da cabeça no uso do laptop e $1,75^{\circ}$ no uso do computador de mesa, além de $57,4^{\circ}$ para flexão de pescoço no laptop e $50^{\circ}$ no computador de mesa, enquanto obtivemos $57,79^{\circ}$ e $47,42^{\circ}$, respectivamente. Donoghue et al. (2013) em estudo com laptops obteve $12,7^{\circ}$ para flexão-extensão do punho, e também Gold et al. (2012) encontrou $-12,79^{\circ}$ para flexão-extensão do punho e $39,52^{\circ}$ para flexão de ombro, comparados a $-13,74^{\circ}$ e $37,79^{\circ}$ obtidos neste estudo de caso.

Com relação aos valores encontrados para ângulo de visão, Chaffín, Andersson e Martin (1999), afirmam que a linha normal de visão situa-se entre 10 a $15^{\circ}$ abaixo da linha horizontal. Esta linha normal de visão é a posição de repouso dos olhos, sendo recomendado que os objetos a serem visualizados fiquem neste limite. Ainda segundo lida (2005) na postura sentada com o tronco ereto, as pessoas preferem visualizar objetos a $20^{\circ}$ abaixo da linha horizontal. Neste estudo, para o sujeito analisado, em todas as condições o ângulo de visão ultrapassa $20^{\circ}$ abaixo da linha do horizonte, entretanto, no caso do computador de mesa, ultrapassa apenas $1,18^{\circ}$, já para o laptop esse valor é maior, ultrapassando em torno de $8^{\circ}$ na condição $\mathrm{N}^{\circ} 2$ e $16^{\circ}$ na condição $\mathrm{N}^{\circ} 3$.

Segundo lida (2005) a flexão de pescoço deve ser até $20^{\circ}$. Acima de $30^{\circ}$ as dores no pescoço começam a aparecer. De acordo com Thornton (1978) o valor ideal de conforto seria $24^{\circ}$. Novamente, em todas as condições o valor ultrapassa o ideal, sendo que na condição $\mathrm{N}^{\circ} 1$ o valor fica em torno de $20^{\circ}$ acima do recomendado, mas na condição $\mathrm{N}^{\circ} 2$ e $\mathrm{N}^{0} 3$ esse valor ultrapassa o recomendado em $33^{\circ}$ e $51^{\circ}$ respectivamente. Já a inclinação do tronco recomendada é entre 90 e $120^{\circ}$ (IIDA, 2005) sendo que todos as condições se enquadram no valor recomendado.

Gerr et al. (2004) afirmam que um desvio ulnar do punho maior que $20^{\circ}$ é significativamente associado a lesões musculoesqueléticas. No caso do computador de mesa (condição $N^{0} 1$ ) o desvio ulnar do punho é de $18,52^{\circ}$, entretanto no caso do laptop, o desvio ulnar é maior que $20^{\circ}$ tanto na condição $\mathrm{N}^{\circ} 2\left(24,35^{\circ}\right)$ como na condição $\mathrm{N}^{\circ} 3\left(28,14^{\circ}\right)$, sendo a condição $\mathrm{N}^{\circ} 3$ a mais problemática.

Mesmo utilizando a mesma configuração de trabalho, observa-se valores divergentes entre o uso do laptop e do computador de mesa, com o último apresentando valores mais próximos de uma posição neutra e consequentemente um menor risco de queixas musculoesqueléticas.

\section{CONCLUSÃO}

O objetivo deste estudo foi determinar e comparar as diferenças ergonômicas no uso de computadores de mesa e laptops, verificando as posturas no plano sagital e transversal envolvidas durante a execução de uma tarefa de digitação e edição em ambos os equipamentos. A análise dos ângulos posturais médios assumidos durante a execução das tarefas com um computador de mesa demonstrou uma postura mais erguida da cabeça e do pescoço e uma postura mais neutra dos ombros, cotovelos e punho em comparação com os resultados posturais durante o uso do laptop.

Os valores obtidos assemelham-se com a literatura pesquisada, evidenciando que o uso de laptop em comparação com o computador de mesa representa um risco maior de queixas musculoesqueléticas, apesar de ser o mais usado e vendido atualmente (GOLD et al., 2012), o que leva a necessidade de mais análises sobre o futuro do trabalho com computadores cada vez menores.

Como características dos computadores analisados, destaca-se os componentes externos, como monitor, teclado e mouse, que por permitirem uma maior flexibilidade de configuração espacial, facilitam o ajuste pessoal do usuário para posturas mais neutras. Apesar dos laptops permitirem uma integração com componentes externos, o fator mobilidade acaba 
exercendo uma forte influência na escolha de um local adequado para sua utilização. Como foi observado no estudo de caso, o seu uso em locais não apropriados como poltronas resulta em posturas menos neutras e um maior risco de queixas.

Sendo assim, conclui-se que apesar dos laptops permitirem uma mobilidade maior, não seriam adequados para períodos de uso contínuo, sendo que para este caso indica-se o uso de computadores de mesa. Este estudo analisou as posturas no laptop sem considerar componentes externos como mouse e teclado, portanto, recomenda-se como estudos futuros a análise postural em laptops com o auxílio de componentes externos, para verificar a influência do display dos laptops em assumir posturas menos neutras.

\section{REFERÊNCIAS BIBLIOGRÁFICAS}

ABERGO. Norma ERG BR 1002 - Código de Deontologia do Ergonomista Certificado. In: http://www.abergo.org.br/arquivos/norma_ergbr_1002_deontologia.pdf.

ASUNDI, K.; ODELL, D.; LUCE, A.; DENNERLEIN, J. Changes in posture through the use of simple inclines with notebook computers placed on stardard desk. Appl Ergon., v.43, p. 4007, 2012.

AYDENIZ, A.; GURSOY, S. Upper extremity musculoskeletal disorders among computer users. Turk J Med Sci., v. 38, p. 235-38, 2008.

BERKHOUT, A.L.; HENDRIKSSON-LARSEN, K.; BONGERS, P. The effect of using a laptopstation compared to using a standard laptop PC on the cervical spine torque, perceived strain and productivity. Appl Ergon., v.35, n.2, p.147-52, 2004.

CASTELLUCCI, I.; ZUÑIGA BENITEZ, L. Postura, disconfort y productividad durante la ejecución de tareas de mecanografía en computadores personales portátiles tipo netbook, con y sin modificaciones ergonómicas. Bol Not PI., v.21, p.1-9, 2013.

CERVO, A.L.; BERVIAN, P.A.; SILVA, R. Metodologia científica. 6 ed. São Paulo: Pearson Prentice Hall, 2007.

CHAFFIN, D.B.; ANDERSSON, G.B.; MARTIN, B.J. Occupational Biomechanics. 3 ed. New York e Toronto: John Wiley \& Sons, 1999.

CONLON, C.F.; REMPEL, D.M. Upper extremity mononeuropathy among engineers. J Occup Environ Med., v.47, p.1276-84, 2005.

CONSELHO NACIONAL DE SAÚDE. Resolução No 196, de 10 de outubro de 1996. In: http://conselho.saude.gov.br/docs/Resolucoes/Reso196.doc.

DONOGHUE, M.; O'REILLY, D.; WALSH, M. Wrist postures in the general population of computer users during a computer task. Appl Ergon., v.44, p.42-7, 2013.

GERR, F.; MARCUS, M.; MONTEILH, C. Epidemiology of musculoskeletal disorders among computers users: lesson learned from the role of posture and keyboard use. J Electro Kines., v.14, p.25-31, 2004.

GERR, F.; MONTEILH, C.P.; MARCUS, M. Keyboard use and musculoskeletal outcomes among computer users. J Occup Rehabil., v. 13, n.3, p. 265-77, 2006.

GIL, A.C. Como elaborar projetos de pesquisa. 3 ed. São Paulo: Atlas, 1996.

GOLD, J.; DRIBAN, J.; YINGLING, V.; KOMAROFF, E. Characterization of posture and comfort in laptop users in non-desk settings. Appl Ergon., v.43, p.392-99, 2012.

HOROWITZ, J. Crippled by computers, Time Magazine, October 12, 1992.

IIDA, I. Ergonomia - Projeto e Produção. São Paulo: Edgar Blutcher, 2005.

JONAI, H.; VILLANUEVA, M.; TAKATA, A.; SOTOYAMA, M.; SAITO, S. Effects of the liquid crystal display tilt angle of a notebook computer on posture,muscle activities and somatic 
complaints. Int J Ind Ergon., v.29, p.219-29, 2002.

PUNNETT, L.; BERGQVIST, U. Visual Display Unit Work and Upper Extremity Musculoskeletal Disorders. A review of epidemiological findings. National Institute of Working Life, 17184 Solna Sweden Ergonomic Expert Committee Document No 1, 1997.

SAITO, S.; MIYAO, M.; KONDO, T.; SAKAKIBARA, H.; TOYOSHIMA, H. Ergonomic evaluation of working posture of VDT operation using personal computer with flat panel display. Ind Health., v.35, n.2, p.264-70, 1997.

SEGHERS, J.; JOCHEM, A.; SPAEPEN, A. Posture, muscle activity and muscle fatigue in prolonged VDT work at different screen height settings. Ergonomics., v.46, n.7, p.714-30, 2003.

SOMMERICH, C.M.; STARR, H.; SMITH, C.A.; SHIVERS, C. Effects of notebook computer configuration and task on user biomechanics, productivity, and comfort. Int J Ind Ergon., v.30, p.7-31, 2002.

STRAKER, L.; JONES, K.J.; MILLER, J. A comparison of the postures assumed when using laptop computers and desktop computers. Appl Ergon., v.28, n.4, p.263-68, 1997.

TITTIRANONDA, P.; REMPEL, D.; ARMSTRONG, T.; BURASTERO, S. Workplace use of an adjustable keyboard: adjustment preferences and effect on wrist posture. Am Ind Hyg Assoc J, v.60, p.340-48, 1999.

TRIVIÑOS, A.N.S. Introdução à pesquisa em ciências sociais: a pesquisa qualitativa em educação. São Paulo: Atlas, 2006.

THORNTON, W. Anthropometric changes in weightlessness. In: McCORMICK EJ, SANDERS MS. Human factors in engineering and design. New York: Mc Graw-Hill Book Co., 1978; 453-82.

VILLANUEVA, M.B.; JONAI, H.; SAITO, S. Ergonomic aspects of portable personal computers with flat panel displays (PC-FPDs): evaluation of posture, muscle activities, discomfort and performance. Ind Health., v.36, n.3, p.282-89, 1998.

VILLANUEVA, M.B.; SOTOYAMA, M.; JONAI, H.; TAKEUCHI, Y.; SAITO, S. Adjustments of posture and viewing parameters of the eye to changes in the screen height of the visual display terminal. Ergonomics., v.39, n.7, p.933-94, 1996. 